\title{
Regeneración forestal tras un incendio: complejidad y protocolos en una aproximación STEM transversal
}

\author{
Manuel García Piqueras iD \\ Facultad de Educación e IES Tomás Navarro Tomás. Albacete.España.manuel.gpiqueras@uclm.es
}

\author{
María Sotos Serrano \\ Facultad de Educación.Albacete.España.maria.sotos@uclm.es
}

[Recibido: 14 Enero 2020. Revisado: 12 Abril. Aceptado: 23 julio de 2020]

\begin{abstract}
Resumen: El objetivo de este artículo es ofrecer una experiencia que implementa prácticas STEM (Science, Technology, Engineering, Mathematics) con alumnado de 12 y 13 años en un escenario de cambio climático global. Se emplea una metodología de Aprendizaje Basada en Proyectos (ABP) mediante una investigación sobre la regeneración vegetal tras un incendio de alta severidad que emplea teledetección y trabajo de campo. La experiencia ilustra cómo dotar de contexto a las asignaturas implicadas, mostrar las interdependencias existentes entre disciplinas y modelizar sistemas tan complejos como los ecosistemas. Los objetivos pivotaron sobre la adquisición de competencias STEM y la conciencia social sobre el cambio climático. En cuanto a la implementación de la experiencia, se especifica el conjunto de actuaciones y protocolos desarrollados en el ámbito de la colaboración entre la enseñanza secundaria y superior.
\end{abstract}

Palabras clave: Regeneración forestal; Cambio climático; Modelización; Aprendizaje Basado en Proyectos; Trabajo en equipo.

Forest regeneration following forest fire: complexity and protocols in a transversal STEM approach

Abstract: The aim of this paper is to provide an experience which implements STEM (Science, Technology, Engineering, Mathematics) practices with 12 and 13 year old students in a global climate change scenario. We present a Project-Based Learning (PBL) methodology through a research project on the regeneration of vegetation after a high-severity fire using remote sensing and field work. The experience illustrates how to provide a context for the subjects involved, shows interdependencies between disciplines and models systems as complex as ecosystems. The objectives focus on STEM skills and social awareness about climate change. Regarding the implementation of the experience, we specify the set of actions and protocols developed within the collaboration between secondary and higher education.

Keywords: Forest regeneration; Climate change; Modelization; Project Based Learning; Teamwork.

Para citar este artículo: Garcia-Piqueras, M., Sotos Serrano, M. (2021) Regeneración forestal tras un incendio: complejidad y protocolos en una aproximación STEM transversal. Revista Eureka sobre Enseñanza y Divulgación de las Ciencias 18(1), 1201. doi: 10.25267/Rev_Eureka_ensen_divulg_cienc.2021.v18.i1.1201

\section{Introducción}

En el mundo actual, donde la ciencia y la tecnología desempeñan un papel fundamental, la disminución del interés por las materias y las carreras científico-tecnológicas tiene un fuerte impacto sobre la economía (Quigley y Herro 2016, European Comission 2015, Cebrián y Junyent 2015). En respuesta a este deseo de fortalecer la economía y atraer al alumnado al ámbito de las ciencias ha surgido, en los últimos años, el movimiento educativo STEM, acrónimo de los términos en inglés de Science, Technology, Engineering, Mathematics. La educación STEM ha sido identificada como una plataforma válida para desarrollar competencias y habilidades sociales muy valoradas tanto a nivel personal como profesional: investigación científica, resolución de problemas, pensamiento crítico y creativo, iniciativa empresarial, colaboración, trabajo en equipo, comunicación y la actitud positiva ante el error; las destrezas meramente técnicas son de poco valor si no están en combinación con las 
competencias y habilidades sociales (Heckman y Kautz 2012, Quigley, Herro, King y Plank 2020).

Existe cierto consenso sobre el desarrollo de competencias y habilidades como un componente y un resultado de la educación STEM (Falloon, Hatzigianni, Bower, Forbes y Stevenson 2020, Balgopal 2020). Sin embargo, el modo en que se consigue implementar no está claramente delimitado, puesto que STEM no puede identificarse con una metodología específica (Couso 2017). Consideraremos que el término STEM idenfitica una plataforma, en constante crecimiento, que involucra herramientas tecnológicas, orientaciones pedagógicas y metodologías. En el ámbito de los instrumentos tecnológicos entraría, entre otros, el empleo de datos remotos, como teledetección o geolocalización (Domènech-Casal 2014, López, Couso, Simarro, Garrido, Grimalt-Alvaro, Hernandez y Pinto 2017).

Respecto a las orientaciones pedagógicas distinguiremos tres ejes: Inclusión, Creatividad y Ciudadanía (Domènech-Casal, Lope y Mora 2019). El eje de la inclusión busca atraer a los estudiantes hacia el ámbito científico-tecnológico, dotándoles de capacidades y habilidades, además de buscar el concurso de alumnado en riesgo de exclusión social o en condiciones socio-económicas inferiores. La creatividad se potencia mediante investigaciones o estudios realizados con el fin de explorar o predecir un determinado fenómeno, así como la integración del Arte en el ámbito científico-tecnológico. La ciudanía se trata, por ejemplo, a través de Controversias Socio-Científicas o la Educación Ambiental, entre otras (Domènech-Casal et al 2019).

En cuanto a las metodologías empleadas cabe destacar el Aprendizaje Basado en Proyectos (ABP) donde el énfasis no se hace sobre conceptos y procedimientos, sino en la resolución de problemas en contextos reales (Slough y Milam 2013). Esta metodología didáctica distingue 4 categorías: Obtener un conocimiento, Disfrutar de una experiencia estética, Resolver un problema y Elaborar un producto (Kilpatrick 1918, Domènech-Casal et al 2019). En esta última categoría el alumnado se fija un objetivo externo al aprendizaje. Este posicionamiento se combina adecuadamente con la educación STEM y enlaza con el ABP de modo que el aprendizaje de las materias en el ámbito científico-tecnológico utiliza una situación del mundo real como estructura central para la enseñanza (King 2012, Sanmartí y Márquez 2017, Holmlund, Lesseeig y Slavit 2018).

En otro orden de cosas, desde un punto de vista organizativo, la teoría de los valores enfrentados (Quinn y Rohrbaugh 1983, Quinn 1988) determina que la efectividad organizativa se caracteriza por dos dimensiones:

1) La estructura organizativa: define la preferencia por la adaptabilidad y flexibilidad frente a la estabilidad y el control.

2) El foco organizativo: sitúa el énfasis en el bienestar y desarrollo de los participantes (interno) frente a la prosperidad de la propia organización (externo).

Así, el marco teórico recoge 4 modelos principales sobre teoría organizativa y de gestión (Quinn 1988): a) relaciones humanas, flexible y enfocado internamente, b) sistemas abiertos, flexible y orientado externamente, c) objetivos racionales, enfatiza el control y lo externo y d) procesos internos, busca el control y subraya lo interno.

Para cada uno de estos modelos se definen distintos tipos de liderazgos: a) facilitador y mentor, b) innovador y broker, c) productor y director, d) monitor y coordinador (Quinn 1988). En particular, dentro del modelo de procesos internos, la figura del coordinador prioriza la estabilidad, el control y la continuidad del proyecto en un entorno de gestión investigadora multidisciplinar (König et al 2013). Múltiples autores (Geoghegan y Dulewicz 2008, Schmid y Adams 2008, Larson y Gray 2014) han puesto de manifiesto la importancia del liderazgo en el éxito gestor. 
Un liderazgo efectivo crea un entorno de trabajo en equipo donde sus integrantes son animados a participar, crecer, aprender y trabajar juntos para conseguir el objetivo propuesto (Novo, Landis y Haley 2017).

\section{Punto de partida. Motivación}

Una de las cuestiones que preocupa en la actualidad a la ciudadanía en general y al alumnado en particular es el cambio climático que dibuja un escenario catastrófico para un futuro relativamente cercano (Asadnabizadeh 2019). En concreto el calentamiento global es una idea recurrente. La degradación de los bosques es una fuente significativa de gases de efecto invernadero, de modo que su control podría tener un importante impacto (Pearson et al 2017).

Por otra parte, un tipo de alumnado matriculado en el IES ${ }^{1}$ Tomás Navarro Tomás tiene una situación socioeconómica baja o, incluso, se encuentra en riesgo de exclusión social.

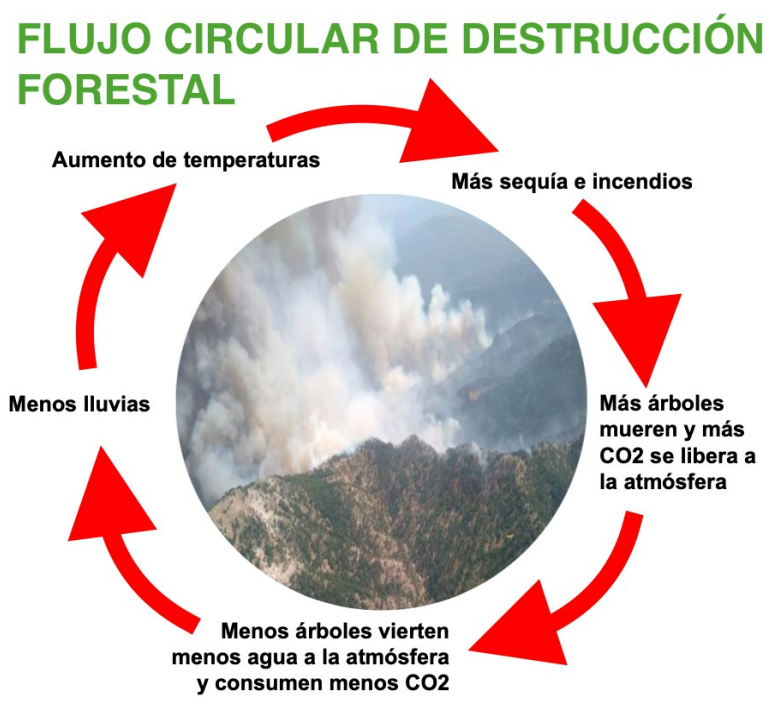

Figura 1. Flujo Circular de Destrucción Forestal: relación del ciclo del dióxido de carbono con el cambio climático y los incendios.

El centro tiene una relación importante con el campus universitario y el parque científicotecnológico de Castilla-La Mancha al estar situado en la misma zona geográfica. Esto facilita, entre otras ventajas, la existencia de profesorado y personal compartido. Un grupo de profesores universitarios y de secundaria relacionados con el IES consideró actuar respecto a ambas problemáticas, tanto la climática como la de exclusión social, de modo que se pudiera aprovechar la conexión existente con la universidad. Así, se decidió implementar una experiencia STEM con alumnado del IES, en colaboración con la Universidad de Castilla-La Mancha (UCLM) y en un contexto de estudio de cambio climático. Se determinó trabajar en este tema con dos grupos, uno de 12 años, con 13 estudiantes (seis mujeres), y otro de 13 años, con 11 estudiantes (nueve mujeres), del IES Tomás Navarro Tomás. En una primera reunión del profesorado de secundaria con el alumnado se comentaron los últimos datos disponibles sobre el Flujo Circular de Destrucción Forestal (figura 1); emisiones de $\mathrm{CO}_{2}$, calentamiento global, sequía y aumento de incendios tanto en frecuencia como intensidad (ESA 2019a). El alumnado tenía un conocimiento aproximado de la relación existente entre las diferentes emisiones de gases de efecto invernadero, como el $\mathrm{CO}_{2}$, a la atmósfera. A cada uno de los grupos se le pidió que expusieran sus opiniones al respecto. Entre todos los comentarios realizados destacamos, por ejemplo, las siguientes ideas preconcebidas:

1. Todos los incendios son malos: desconocían que el fuego es un mecanismo propio del ecosistema que favorece la germinación de ciertas semillas; no identificaban que lo realmente grave es el acortamiento de los periodos de repetición y su intensidad.

2. El incendio únicamente deja secuelas en la vegetación: no describían el daño que podía ejercer en el suelo, como la pérdida de nutrientes y la erosión que facilitan el avance de la desertificación.

\footnotetext{
${ }^{1}$ Las siglas IES se refieren a Instituto de Educación Secundaria.
} 
3. El incendio afecta a todas las zonas por igual: no contemplaban la existencia de zonas más vulnerables que otras. Por ejemplo, en igualdad de condiciones, las regiones cuya orientación es principalmente de umbría tienen una mayor capacidad de regeneración que aquellas situadas en la solana.

El alumnado comentó, a nivel particular, distintas experiencias que habían tenido con los incendios y la discusión desembocó en un gran incendio acaecido en 2017 en el suroeste de la provincia de Albacete (España), entre las localidades de Riópar y Molinicos (Montero-Silvestre 2018). Se decidió estudiar este caso aunque quedara relativamente lejos del centro $^{2}$. El profesorado intervino para comentar la posibilidad de participar en el concurso internacional Climate Detectives, patrocinado por la Oficina Europea de Recursos para la Educación Espacial (ESERO), donde había que reconocer un problema climático del entorno, tomar datos, analizarlos y elaborar conclusiones.

\section{Diseño global de la experiencia}

La investigación sobre la evolución de una zona incendiada es un problema abierto donde hay una multitud de variables involucradas. Hay que tener en cuenta que la parte del ecosistema afectada por el incendio era muy cambiante y no estaba aislada, pues mantenía un contacto continuo con su entorno. A la dificultad de la investigación se añadía que los recursos económicos de los que podíamos disponer eran limitados. Se dispuso elaborar un plan de actuaciones formativas que deberían contribuir a la consecución de los siguientes objetivos:

1) Promover la adquisición de competencias y habilidades STEM, incluyendo la metodología científica, la recogida de datos, visualización y análisis.

2) Mejorar la concienciación y el conocimiento del clima del planeta, tanto a nivel local como global, así como prepararlos para los futuros retos climáticos.

La participación en la fase final ${ }^{3}$ de Climate Detectives requería la elaboración de un informe final y un vídeo explicativo de tres minutos, como máximo, que cumplieran los siguientes objetivos:

1) Identificar un problema climático local mediante la observación de su entorno local e investigarlo mediante la utilización de datos de campo o teledetección.

2) Realizar propuestas, en base a la investigación realizada, para reducir el problema.

El informe y el vídeo serían el producto final de un proyecto STEM-ABP categorizado como Elaborar un producto, mencionado en la introducción. En este marco se decidió subdividir el trabajo en pequeñas tareas que fueron proporcionadas sucesivamente al alumnado, teniendo como referencia los contenidos presentes en el currículum oficial de Biología, Matemáticas, Física y Química, Geografía e Historia y Tecnología. El grupo de 12 años cursó Biología, pero no Física y Química, mientras que el de 13 años no tenía Biología como asignatura, pero sí Física y Química.

La recogida de datos sobre cómo está aprendiendo el alumnado, su análisis y la toma de decisiones, nos servirá como evaluación, es decir, como regulación del proceso de aprendizaje (Sanmartí 2020). Dicha recogida se estableció mediante el diálogo constante entre el profesorado y el alumnado sobre los asuntos tratados en cada actividad. Tanto el profesorado como el alumnado analizaban y decidían sobre el proceso de enseñanza-aprendizaje (evaluación formativa y formadora). Esta regulación permitió establecer ciertos límites, en

\footnotetext{
${ }^{2}$ En torno a unos 120 kilómetros por carreteras secundarias.

${ }^{3}$ Para participar en la iniciativa Climate Detectives no era necesario realizar ningún informe, pero sí era un requisito para acceder a una fase final donde se reconocieran distintos proyectos mediante la concesión de premios. Los tres primeros equipos tendrían la oportunidad de ser invitados al Living Planet Symposium en Milán (2019) para explicar sus investigaciones.
} 
consonancia con la teoría de la zona de desarrollo próximo de Vygotsky, i.e., los objetivos de aprendizaje deberían situarse entre nivel de desarrollo efectivo del estudiante y el nivel de desarrollo potencial (Salkind 2004). Por ejemplo, cuando una actividad resultaba muy difícil de completar o era tan fácil que resultaba pesada y repetitiva, se buscaban alternativas a sugerencia del profesorado o del alumnado. A menudo se proponían una serie de técnicas, herramientas, pequeñas investigaciones o ideas en general, que luego se descartaban o postergaban en favor de otras, fruto de la discusión continua entre el alumnado y el profesorado. Era habitual que surgieran gran cantidad de ideas, sin embargo, muy pocas eran viables para su desarrollo. Por lo que respecta a las calificaciones, se decidió que cada asignatura empleara estándares de aprendizaje propios de la disciplina a criterio del profesorado encargado.

En los párrafos siguientes se trata de resumir el camino recorrido en todo este proceso, indicando cuáles fueron algunos de los momentos clave.

\section{Actuaciones: planificación y temporalización. El papel del coordinador}

La estructura organizativa del proyecto involucraba la educación secundaria y universitaria, y estaría enfocada hacia el equilibrio, mientras que el foco organizativo estaría orientado hacia el bienestar de sus integrantes. Así, el proyecto se catalogó en procesos internos, uno de los 4 modelos sobre teoría organizativa mencionados en la introducción. En particular, un liderazgo basado en la figura del coordinador, en el sentido de König (2013) y Quinn (1988), dotaría al proyecto de estabilidad, control y continuidad. Se consideró oportuno que dicha figura recayera en un profesor que impartía clases al alumnado del IES Tomás Navarro Tomás y que también era profesor universitario de la Facultad de Educación de Albacete (UCLM). Además, había sido impulsor de la iniciativa desde el principio y estaba formado en competencias STEM por parte de ESERO.

El trabajo se desarrolló durante el curso 2018-19, desde septiembre de 2018 hasta abril de 2019. Distinguiremos, principalmente, dos tipos de actuaciones: charlas y seminarios. Las charlas eran actuaciones de una o dos sesiones de unos 50 minutos, donde hay un mayor peso teórico, mientras que los seminarios duraban una jornada completa y se trabajaban aspectos prácticos fundamentalmente. En ocasiones, las actividades planteadas necesitaban de más tiempo para su finalización, entonces se convocaba al alumnado a sesiones de refuerzo, generalmente en el propio IES. Todo el alumnado implicado participó en todas las actuaciones ${ }^{4}$.

Las sesiones que se llevaron a cabo en la universidad fueron dirigidas por profesorado universitario del centro donde se desarrollaron: Facultad de Educación de Albacete o Escuela Técnica Superior de Ingenieros Agrónomos y de Montes (ETSIAM) de Albacete (UCLM). El IES Tomás Navarro Tomás cuenta con dos ventajas principales: (1) cercanía al campus universitario, de modo que no suponía una pérdida de tiempo significativa el desplazarse a las facultades mencionadas; y (2) profesorado que trabaja como asociado en distintas facultades, permitiendo establecer un ambiente de cooperación y confianza mutua. De hecho, el IES tenía firmados dos convenios de colaboración con ambas facultades que se traducían en ventajas para todas las partes implicadas. Así, por ejemplo, el alumnado podía asistir a ciertos eventos universitarios, mientras que las facultades se promocionaban ante un posible futuro alumnado.

Al margen de entidades estrictamente educativas, colaboraron el Ayuntamiento de Albacete, con dotación económica, y el Instituto Técnico Agronómico Provincial de Albacete (ITAP), mediante una visita guiada y una charla.

\footnotetext{
${ }^{4}$ Salvo por alguna cuestión personal.
} 
En todas las actuaciones siempre participaba el coordinador del proyecto. Generalmente siempre le acompañaba otro profesor del IES que impartía clase al alumnado y también participaba en el proyecto. Las actuaciones eran planificadas y consensuadas previamente entre el profesorado universitario y el de secundaria. El profesorado de secundaria disponía de una sesión semanal para desarrollar el proyecto. El profesorado universitario concertaba reuniones con el coordinador del proyecto a petición de éste. Cabe resaltar que el coordinador desempeñó un papel crucial como canalizador de las distintas sensibilidades y criterios sobre la forma de conseguir los objetivos propuestos de todo el profesorado implicado, así como de toda la comunidad educativa, de modo que todas las actuaciones se ajustaran a lo previsto.

El coordinador se encargaba de informar, con suficiente antelación, de las futuras actuaciones previstas a todos los colectivos implicados (equipo directivo, familias, profesorado, entre otros).

Las principales actuaciones se distribuyeron de la siguiente manera:

1) Discusión inicial sobre el cambio climático (10 de octubre de 2018, IES Tomás Navarro Tomás).

2) Charla I (24 de octubre de 2018, Facultad de Educación de Albacete): Trabajo en equipo y gestión de conflictos.

3) Charla II (21 de noviembre de 2018, Facultad de Educación de Albacete): La luz y su espectro. Índices espectrales 5 .

4) Seminario I (16 de enero de 2019, ETSIAM): Cálculo de índices espectrales en Sentinel-2 y Landsat ${ }^{6}$. Establecimiento del perímetro del incendio en Google Earth y generación de mapas con información geográfica enriquecida (extensión '.kmz').

5) Seminario II (17 de enero de 2019, Facultad de Educación de Albacete y trabajo en casa): Obtención de mapas '. $\mathrm{kmz}$ ' asociados a índices espectrales de la zona afectada por el incendio de 2017 (Riópar).

6) Seminario III (25 de enero de 2019, IES Tomás Navarro Tomás y trabajo en casa): Creación del mapa de resistencia regenerativa para la zona afectada por el incendio de 2017. Interpretación de una ficha de recogida de datos en campo.

7) Seminario IV (31 de enero de 2019, ETSIAM). Perímetro incendio Yeste 2017 en parcelas de resistencia regenerativa baja, media y alta: Colecta de datos sobre el terreno mediante fichas destinadas a tal efecto?

8) II Día Ciencia TNT y Jornada de Puertas Abiertas ${ }^{8}$ (14 de febrero de 2019, IES Tomás Navarro Tomás): Los estudiantes explicaron sus investigaciones al alumnado de $6^{\circ}$ de primaria de los colegios invitados. Se explicaron los distintos índices espectrales empleados en el estudio, así como imágenes vía satélite de una localización e interpretación de los resultados obtenidos. También se realizó una labor de concienciación sobre la importancia de la conservación de los bosques, sobre todo de

\footnotetext{
${ }^{5}$ Los índices espectrales son combinaciones de la reflectividad de dos o más longitudes de onda que indican la abundancia relativa de ciertas características. Hay índices diseñados para monitorizar vegetación, mientras que otros son útiles para identificar áreas quemadas, agua o determinadas características geológicas.

${ }^{6}$ Sentinel-2 y Landsat son dos misiones dirigidas por la ESA y EE. UU., respectivamente. En ambos casos gestionan un conjunto de satélites cuyo objetivo es la observación de la corteza terrestre.

${ }^{7}$ La salida estaba prevista para el lunes 28 de enero, pero se tuvo que aplazar porque se anunciaba nieve en el parte meteorológico; finalmente se llevó a cabo durante toda la jornada del jueves jueves 31 de enero.

${ }^{8}$ Se hacían explicaciones en turnos de 15 minutos, de los que reservaban los últimos 5 minutos a cuestiones.
} 
la barrera natural que supone la región de estudio con respecto al avance de la desertificación en el sur de Europa.

9) Charla III (25 de marzo 2019, ITAP): Visita guiada y charla sobre análisis de suelos.

10) Del 25 de marzo hasta el 15 de abril, fecha en que se entregó el producto final, se estableció una reunión semanal de 50 minutos con cada grupo?.

\section{Actuaciones. Investigación y desarrollo}

Dada la importancia del trabajo en equipo se realizó una primera charla que trató los siguientes puntos:

1. Cada persona tiene una serie de cualidades y defectos. Si cada uno de nosotros fuera capaz de canalizar sus virtudes y ponerlas a disposición del equipo, entonces ganaríamos todos. Es una máxima de la teoría de juegos: si colaboramos nos ayudamos, si nos enfrentamos nos anulamos (Peters 2015).

2. El factor humano es muy difícil de gestionar y los conflictos, en ocasiones, son inevitables. Cuando surjan, es conveniente mantener la calma (los insultos y los reproches no hacen más que empeorar las cosas), argumentar con datos objetivos y si al final el acuerdo es imposible, se transmite el problema al profesorado encargado de la actividad para que medie.

A continuación, y antes de realizar trabajo de campo, se decide tratar la observación terrestre mediante datos obtenidos vía satélite y su posterior procesamiento mediante distintos índices espectrales ${ }^{10}$, fundamentalmente el NDVI, un indicador de biomasa fotosintéticamente activa (Weier y Herring 2000), y el NBR, usado para delimitar áreas quemadas en grandes incendios (Keeley 2009). En estos primeros estadios se necesitaba que el alumnado fuese capaz de:

1. Conocer los distintos valores que toma el espectro electromagnético de la luz (Física y Química) para comprender cómo se miden los índices NDVI y NBR.

2. Aplicar la razón y proporción al cálculo de los índices mencionados (Matemáticas).

3. Comprender las fases de la fotosíntesis, los factores que la afectan, su importancia biológica y su relación con el espectro de la luz (Biología y Geología).

4. Leer e interpretar mapas haciendo uso de la escala (Matemáticas y Geografía e Historia). Así como el estudio de regiones y áreas (Matemáticas).

5. Generar, analizar e interpretar gráficas que registren la evolución de los índices espectrales en un determinado período temporal (Matemáticas y Tecnología).

Para un correcto desarrollo del proyecto, era necesario que conocieran los índices NDVI y NBR, de modo que se celebró una segunda charla donde se explicó en qué consistían dichos índices.

A continuación, se desarrolló un primer seminario (figura 2) para tratar cuestiones prácticas relacionadas con el manejo de software específico sobre tratamiento de imágenes vía satélite en $\mathrm{EO}$ Browser ${ }^{11}$ :

\footnotetext{
${ }^{9}$ En ocasiones, se hacían uso de los recreos cuando los 50 minutos no habían sido suficientes.

${ }^{10}$ Se utilizaron los siguientes índices: NDVI (índice de vegetación de diferencia normalizada), NBR (índice de calcinación normalizada), dNDVI (índice de vegetación de diferencia normalizada diferenciada) y dNBR (índice de calcinación normalizada diferenciada) a partir de imágenes de satélite públicas.

${ }^{11}$ EO Browser, https://apps.sentinel-hub.com/eo-browser/, Sinergise Ltd.
} 
1. Ubicación del área de estudio a partir del nombre de la localidad o bien mediante coordenadas geográficas.

2. Obtención de imágenes de satélite para una determinada localidad y un período de tiempo determinado evitando, en la medida de lo posible, la interferencia de las nubes.

3. Tratamiento de la imagen a partir del índice escogido (NDVI, NBR, dNDVI y dNBR).

4. Creación de polígonos que establezcan una región de interés, a partir de los cuáles se obtienen datos del índice estudiado mediante gráficas, así como la

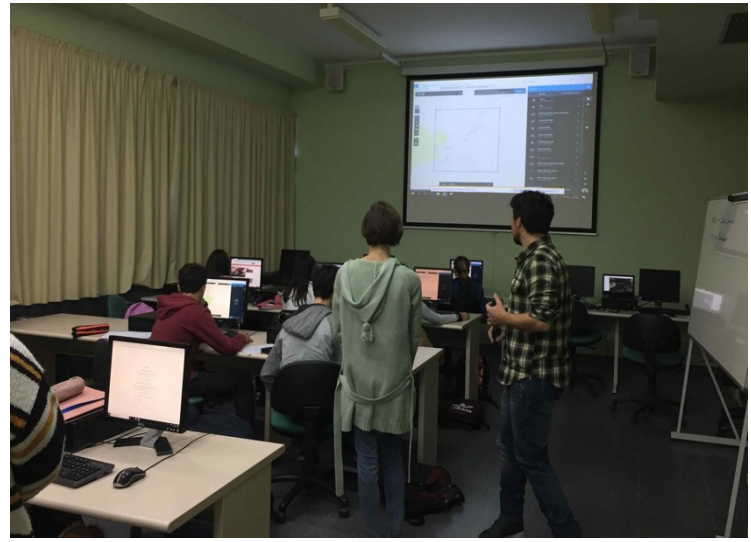

Figura 2. Profesores de la ETSIAM y alumnado del IES Tomás Navarro Tomás durante el seminario de obtención de datos mediante satélites (EOBrowser) aplicados al estudio de la vegetación. inclusión de polígonos ya generados con anterioridad.

5. Exportación de imágenes procesadas en función del correspondiente índice estudiado.

6. Creación de gráficas, a partir de los datos de satélites Sentinel, para plasmar la evolución de un determinado índice.

7. Aplicación de lo aprendido: localización de la zona de estudio (área de Riópar/Yeste/Torre Pedro afectada por el incendio de 2017) y generación de imágenes vía satélite en base a los índices NDVI y NBR.

En un segundo seminario se trabajaron cuestiones técnicas relacionadas con Google Maps (https://www.google.es/maps) y Google Earth (https://www.google.com/earth/), así como una aplicación práctica de lo tratado hasta el momento:

1. Establecimiento del perímetro del incendio, tanto en Google Maps como en Google Earth.

2. Incorporación a Google Earth de la información obtenida vía satélite mediante archivos '.kmz'. Este tipo de ficheros pueden ser generados a partir de imágenes obtenidas mediante EO Browser para un determinado índice.

3. Aplicación de lo aprendido: obtención de los mapas '.kmz' asociados a los índices NDVI y NBR para imágenes de satélite de la zona de estudio durante el período abril 2017 a febrero $2019^{12}$. El período es más amplio que el del incendio, julio-agosto 2017, para enmarcarlo y compararlo con la evolución asociada a la estacionalidad.

Se dedicaron dos sesiones de refuerzo para poner en común el trabajo realizado en grupos. El alumnado plasmó, con datos objetivos, el alto impacto que tuvo el incendio en la zona y cuál fue su evolución: hay zonas muy afectadas que, tras casi dos años, no muestran señales de recuperación, mientras que hay otras que sí parecen mejorar en términos de cobertura vegetal. Los valores obtenidos a partir de la teledetección coincidían con las notas de prensa recopiladas y esto reforzó su confianza en los datos obtenidos vía satélite. A partir de aquí, se les pide estudiar dichos valores con más detalle, pero el alumnado no entendía por qué había que ir más allá.

Se incidió en las distintas causas que podían producir diferentes velocidades de recuperación y se organizó un tercer seminario donde se trataron algunas de las variables que más podrían

\footnotetext{
${ }^{12} \mathrm{El}$ incendio se produjo en julio y agosto de 2017.
} 
influir en la regeneración de la vegetación. Se consideró necesario que el alumnado tuviera claros los siguientes conceptos:

1. Puntos cardinales y orientación geográfica (Matemáticas y Geografía e Historia).

2. Características y reproducción de especies vegetales (Biología y Geología).

3. Características de los suelos (Física y Química, Biología y Geología).

Así, el tercer seminario trató la recuperación vegetal y su relación con las siguientes variables:

1. Pendiente: es una cuestión clave puesto que al desaparecer la cubierta vegetal se pierde suelo. En ocasiones, dicha pérdida se ve agudizada si después del incendio ocurren lluvias torrenciales que arrastran gran cantidad de material, lo cual es característico de esta región.

2. Orientación: las zonas ubicadas en la solana están más expuestas a la fuerza del sol.

3. Suelo: la textura del suelo, es decir, la cantidad y tamaño de sus sustancias inorgánicas (arcilla, limo y arena), así como la concentración de metales pesados y la caliza activa son indicadores de la fertilidad de un suelo.

4. Vegetación: determinadas especies tienen mayor capacidad de resistencia a los incendios y de regeneración que otras.

También se citaron la evolución del régimen pluviométrico, la intensidad de los vientos o el impacto de la despoblación. En este sentido se habló a grandes rasgos de la teoría de la complejidad (Garcia-Piqueras 2017) iniciada en 1686 por Leibniz (2017). Este afirmaba que el universo es inabarcable en toda su vastedad de elementos e interacciones y, sin embargo, es posible, a partir de unas pocas leyes naturales, sintetizar, explicar y predecir su comportamiento. Es así como la ciencia genera un mejor conocimiento del cosmos por medio de modelos teóricos corroborados mediante datos experimentales. El objetivo en esta fase del proyecto consistió en analizar los datos y sintetizarlos en un modelo que sirviera para identificar consecuencias y realizar predicciones. Así, se acordó que las variables 1, 2, 3 y 4, mencionadas anteriormente, eran las más relevantes para modelizar el fenómeno de la regeneración vegetal en nuestro caso particular.

Una vez distinguidos los factores más importantes que inciden en la recuperación vegetal tras un incendio, se programó un cuarto seminario donde el alumnado se dividió en grupos de tres o cuatro personas y cuyos objetivos principales fueron los siguientes:

1. Estudio de un mapa de daños o de resistencia regenerativa (figura 3), como el mapa que predice la resistencia a la regeneración de un área incendiada, realizado por una alumna de la ETSIAM en su Trabajo de Fin de Grado (Montero-Silvestre 2018).

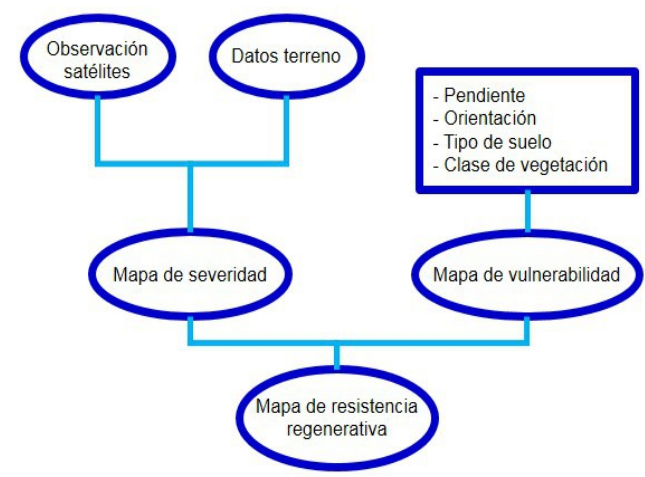

Figura 3. Procedimiento de obtención de un mapa de 'daños' o 'resistencia regenerativa' a partir de las variables consideradas en el proyecto. 


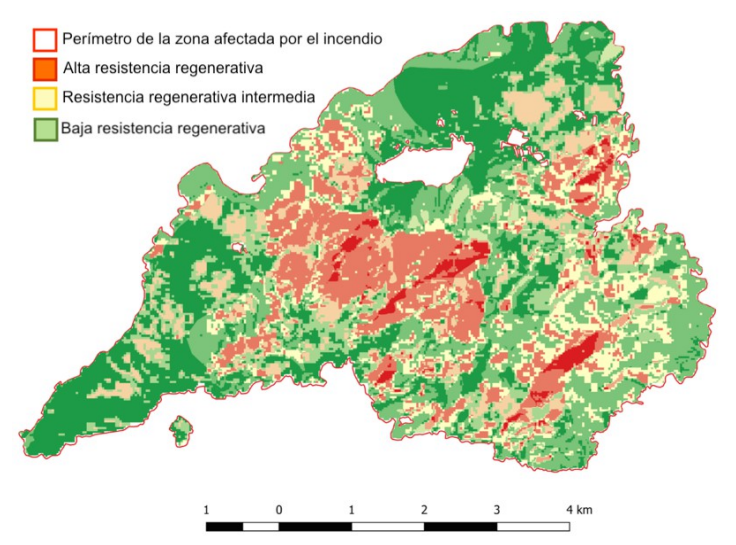

Figura 4. Mapa de resistencia regenerativa restringido a la zona de estudio.

2. Recálculo del mapa de resistencia regenerativa a partir de los pesos ${ }^{13}$ establecidos por el alumnado a las distintas variables consideradas: pendiente, orientación, suelo y vegetación (figura 4). Cada grupo propuso, tras consultar con el profesorado universitario que dirigía el seminario, la incidencia prevista en la regeneración de la inclinación, la orientación, el tipo de suelo y la clase de vegetación. El alumnado acordó darles los siguientes pesos a las variables consideradas: $35 \%$ para la inclinación, 35\% en función de la orientación, 15\% por el tipo de suelo y 15\% por la clase de vegetación. Dichos pesos, aplicados a los mapas de la región de estudio proporcionados por la ETSIAM, produjeron distintas velocidades de recuperación. Se acordó clasificar en tres niveles: alta, media y baja resistencia regenerativa. A mayor resistencia regenerativa peor era la previsión de recuperación.

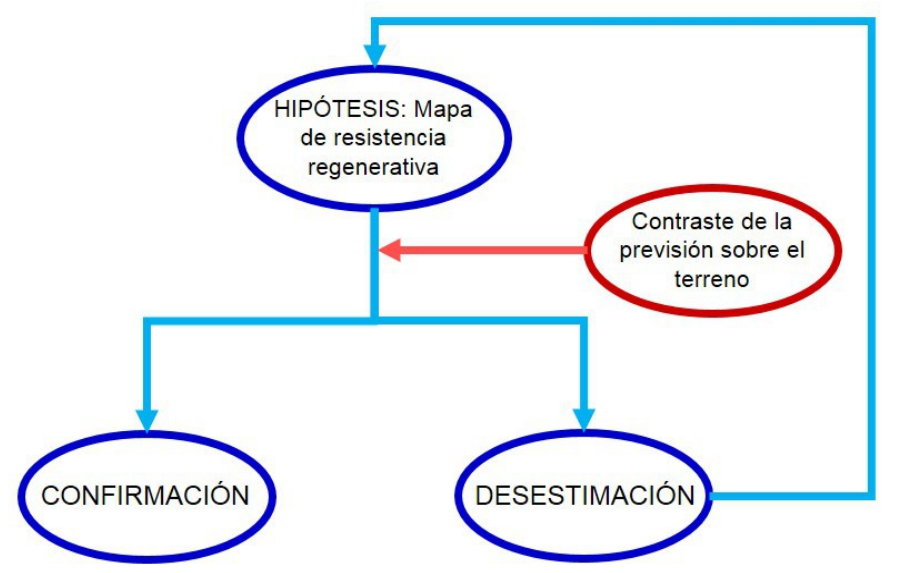

Figura 5. Diagrama de flujo relativa a la predicción elaborada mediante un mapa de resistencia regenerativa y su correspondiente confirmación o desestimación.

Posteriormente, se recordó que los datos han de ser contrastados con el trabajo de campo (figura 5) y se organizó una salida a la zona de estudio. Para ello, se identificaron tres parcelas de 30*30 m.: P12, P211 y P213 ${ }^{14}$, fácilmente accesibles. Cada una de ellas estaba en una zona clasificada como alta, media y baja resistencia regenerativa, respectivamente.

\footnotetext{
${ }^{13} \mathrm{El}$ profesorado de la ETSIAM indicaba que la pendiente solía tener mucha influencia así como la orientación, mientras que el suelo y la vegetación solían tener menos influencia. Posteriormente se llegó a un acuerdo entre todos los grupos tras una discusión muy abierta.

${ }^{14}$ Esta es la nomenclatura manejada por la ETSIAM, pues estas parcelas ya habían sido marcadas previamente y geolocalizadas tras el incendio (Montero-Silvestre 2018).
} 
Antes de llevar a cabo el trabajo de campo, el alumnado debía adquirir los siguientes conocimientos:

1. Clasificación de especies vegetales en hierba, arbusto o árbol, e identificación de especies importantes para el estudio, como el P. Halepensis (Biología y Geología).

2. Manejo de hojas de cálculo para la tabulación de datos e implementación de fórmulas matemáticas que ayuden a sintetizarlos (Tecnología y Matemáticas).

Era importante que el alumnado comprendiera la importancia de la verificación de las hipótesis. Para ello se ofrecieron distintos ejemplos de la historia de la ciencia ${ }^{15}$. El alumnado, en general, se mostraba dispuesto a realizar una excursión a Riópar, pero había una parte reticente a obtener datos sobre el terreno. Este grupo no alcanzaba a ver la importancia del trabajo de campo, pues confiaban exclusivamente en la teledetección para confirmar o desmentir hipótesis objetivamente ${ }^{16}$.

El alumnado se dividió en tres equipos y cada uno recabó datos en las tres zonas catalogadas como baja, media y alta resistencia regenerativa. Estos datos se pusieron después en común entre todos los equipos. El procedimiento escogido fue el Método de intercepción en un rango de vegetación aleatorio (Canfield 1941), el cual describe que, a partir de un punto central, marcado con una estaca, se separan con cinta métrica tres partes iguales del círculo de radio $15 \mathrm{~m}$. centrado en dicho punto central (figura 6).

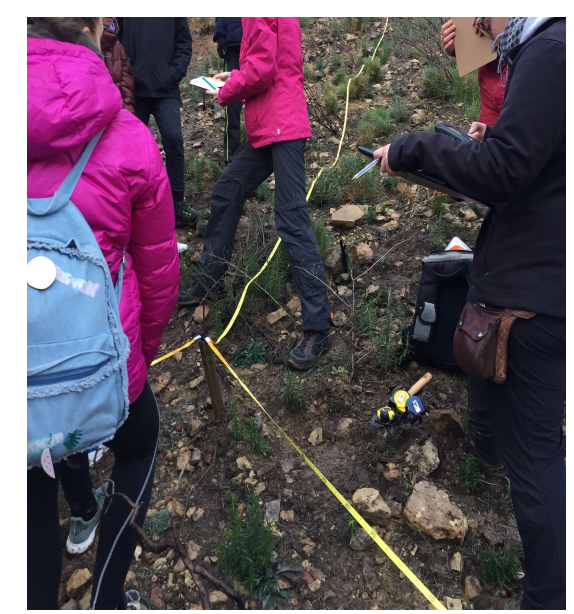

Figura 6. Método de intercepción en un rango de vegetación aleatorio.

A lo largo de cada uno de los tres radios trazados del círculo, se anotó la longitud de la cinta situada encima del suelo desnudo y la longitud de la cobertura vegetal interceptada, así como sus características. Más tarde, se clasificó cada individuo interceptado como hierba, arbusto o árbol, haciendo énfasis en la especie $P$. Halepensis, puesto que es la que compone principalmente los bosques de esta zona. Finalmente, toda esta información fue procesada mediante una hoja de cálculo (figura 7).

\footnotetext{
15 Tales como el experimento del martillo y la pluma de Neil Armstrong que daba la razón a Galileo en su tesis sobre la caída de los cuerpos.

${ }^{16}$ Se indicó que la teledetección puede reconocer como zonas totalmente calcinadas aquellas que, en realidad, solo lo están parcialmente (por ejemplo, cuando solo se queman las copas de los árboles).
} 


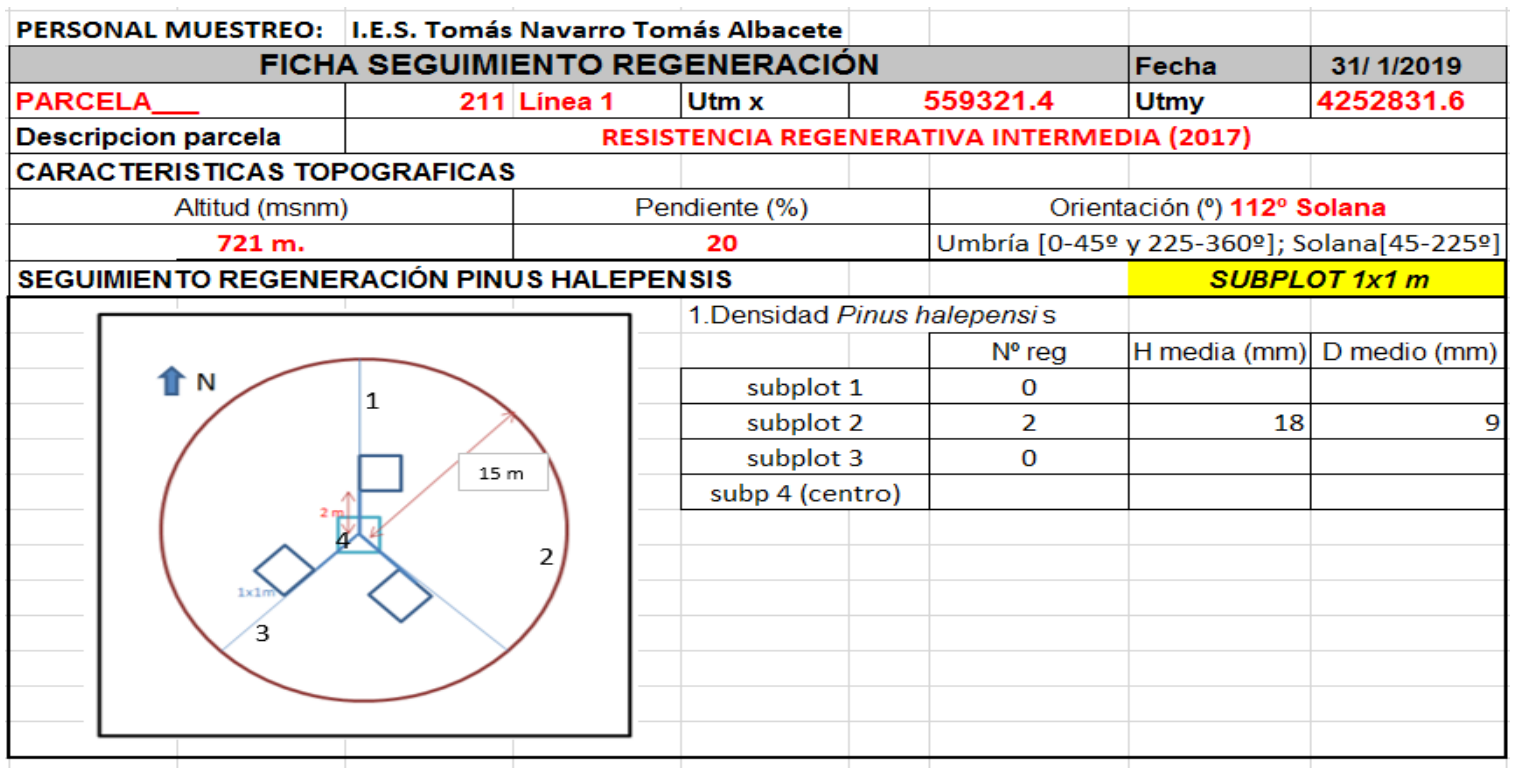

MÉTODO DE INTERCEPCIÓN DE LÍNEAS

LINEA 1 (MAX_Pendiente)

Elemento (sp/piedra/mo/pino quemado/suelo desnudo) Interc $(\mathrm{cm})$

\begin{tabular}{|c|c|}
\hline Elemento $(\mathrm{sp} /$ piedra/mo/pino quemado/sue/o desnudo) & Interc $(\mathrm{cm})$ \\
\hline COBERTURA VEGETAL & $10+15+2+15+15+3+15+5+5$ \\
\hline SUELO DESNUDO & $65+5+60+505+120+290+3$ \\
\hline RIQUEZA & 8 \\
\hline
\end{tabular}

Figura 7. Hoja de cálculo para el registro de la cobertura vegetal en cada parcela.

Adicionalmente, se tomaron muestras de suelo en cada uno de los tres radios para su posterior análisis; este proceso se realizó en las tres parcelas (P12, P211 y P213).

Una vez recogidas las muestras de suelo, el profesorado juzgó oportuno analizarlas en detalle entregando las muestras de suelo recogidas al ITAP (Instituto Técnico Agronómico Provincial) para su análisis. Se descartó que fuera el propio alumnado el que llevara a cabo el análisis de las muestras, debido a la necesidad de emplear instrumental específico no disponible en el centro y por la gran cantidad de tiempo que se necesitaría invertir. Sin embargo, se estimó conveniente que el alumnado conociera el proceso de los análisis sin entrar en excesivos detalles técnicos. Para ello, se organizó una tercera charla en el ITAP, llevada a cabo por los profesionales encargados de realizar dichos análisis. Se trataron los siguientes aspectos:

1. El suelo como parte integrante y clave en la evolución de la vegetación.

2. Enfermedades y plagas típicas de la zona de estudio.

3. Textura del suelo: arena, limo y arcilla a nivel práctico, con muestras reales.

4. Obtención de los principales elementos químicos (tabla periódica) en las muestras, mediante análisis por espectroscopía de absorción atómica (García y Báez 2012).

5. Interpretación y conclusiones a partir de los resultados obtenidos en los análisis.

A partir de aquí se consideró que el alumnado disponía de información suficiente para analizar los datos obtenidos y elaborar conclusiones.

\section{Informe final}

El certamen Climate Detectives pedía un informe que incluyera el proceso de investigación desarrollado (métodos, resultados y conclusiones) empleando un número muy limitado de recursos. Una de las consignas dadas fue la síntesis, de modo que una persona ajena al 
proyecto no tuviera que dedicar una gran cantidad de tiempo en asimilarlo. Se delimitaron los siguientes apartados para el informe final:

1. Objetivos y justificación.

2. Trabajo de campo y análisis de suelo.

3. Observación de la tierra.

4. Conclusiones.

El alumnado se dividió en tres grupos, de modo que cada grupo se encargó de uno de los tres primeros apartados mencionados. Las conclusiones se redactaron a partir de un acuerdo general. El informe consistiría en varios carteles científicos donde se incluyera información visual sobre las principales variables estudiadas (pendiente, orientación, suelo y vegetación), así como el cambio del NDVI a lo largo de las estaciones.

Para la elaboración del cartel sobre Objetivos y justificación (figura 8), el profesorado les proporcionó distintas fuentes para que indagaran sobre el papel de los incendios en la evolución del cambio climático (AEMET 2019a, 2019b). También se incidió en otra consecuencia de los incendios: la pérdida de recursos y la despoblación. Así, se pidió que obtuvieran datos al respecto del Instituto Nacional de Estadística (INE) y elaboraran un gráfico informativo. El grupo encargado estimó conveniente incluir los siguientes puntos:

\subsection{El Flujo Circular de Destrucción Forestal (figura 1).}

1.2. Datos de la Agencia Espacial Europea, que estiman en un $25-35 \%$ el impacto de los incendios en todo el aumento del efecto invernadero interanual (ESA 2019a).

1.3. Recurrencia de incendios en períodos cada vez más cortos de tiempo en la Sierra de Alcaraz, uno de los últimos reductos contra el avance de la desertificación ${ }^{17}$.

1.4. Éxodo rural y despoblación, agravados por la desaparición progresiva de uno de los motores económicos locales: el bosque. Esta cuestión se contempla en diversos planes estratégicos a nivel nacional (Programa Especial de Ciencia, Tecnología e Innovación) y europeo (Research and Innovation Smart Specialisation Strategy) para nuestra área de estudio: sostenibilidad de la producción primaria y de los sistemas forestales, turismo y actividades de ocio rurales (senderismo, montañismo...), así como la salud y el bienestar de las poblaciones rurales en continuo cambio demográfico.

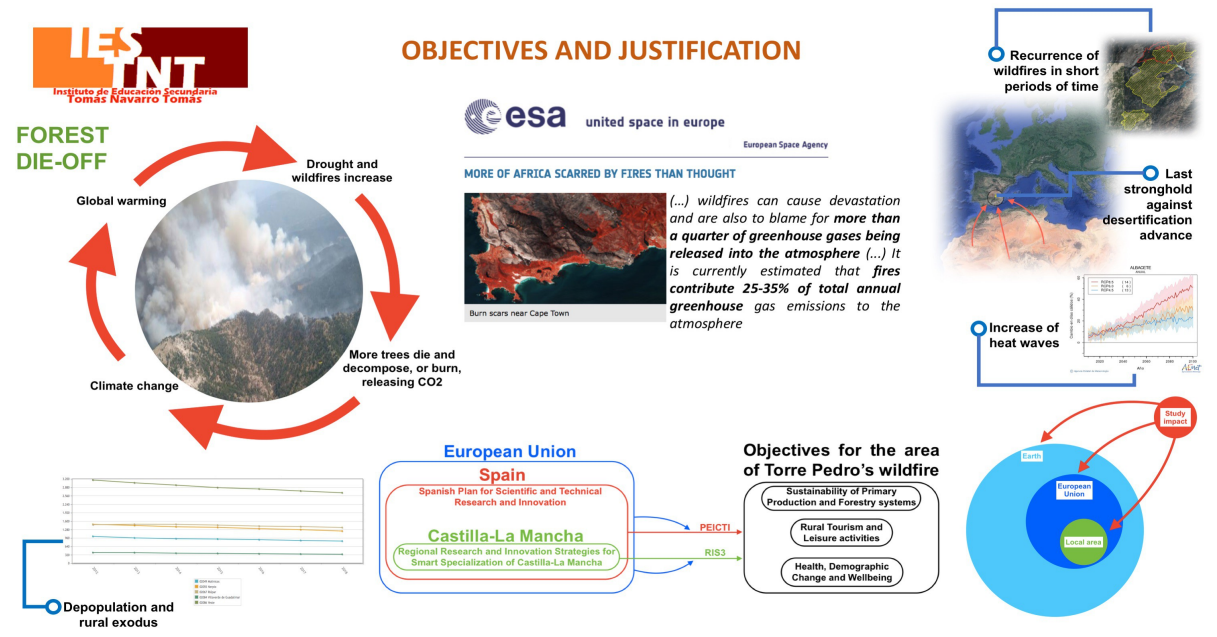

Figura 8. Objetivos y justificación (Cartel 1 del informe final).

\footnotetext{
${ }^{17}$ Gran parte de la zona estudiada había sido ya afectada por otro gran incendio en 1994 solo 23 años antes.
} 


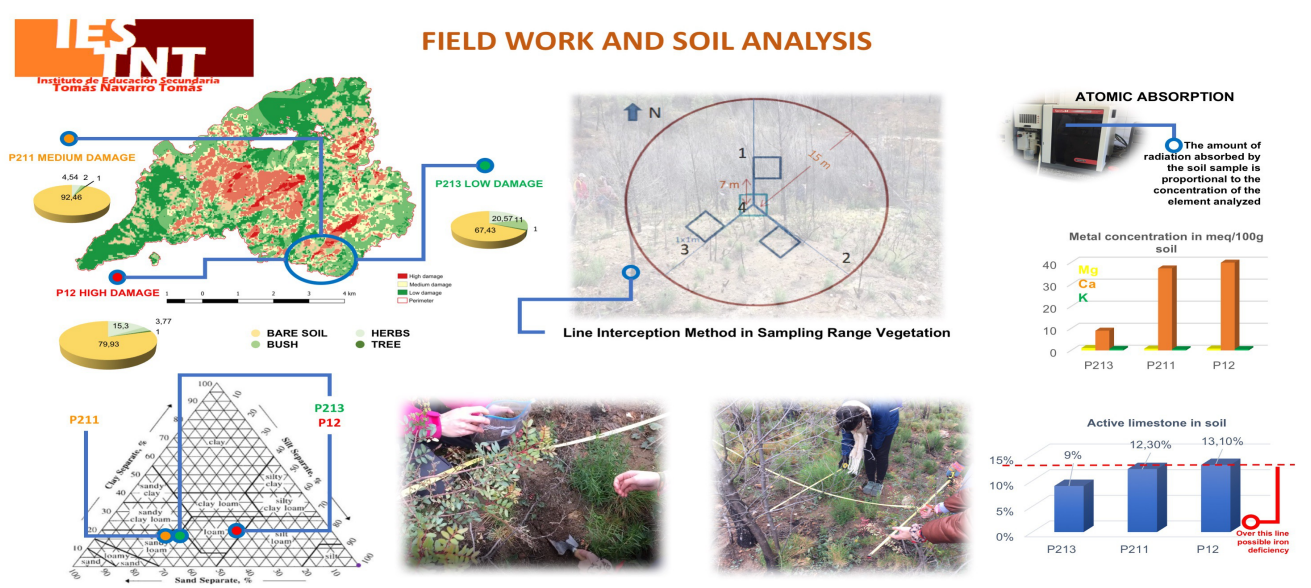

Figura 9. Trabajo de campo y análisis del suelo (Cartel 2 del informe final).

En cuanto al apartado Trabajo de campo y análisis del suelo (figura 9), el grupo encargado analizó los informes del ITAP y las hojas de cálculo con la información de cobertura vegetal para las parcelas estudiadas. Se consideraron los siguientes aspectos:

2.1. El estudio de la cobertura vegetal mostraba un alto porcentaje de suelo desnudo en las tres parcelas estudiadas, seguido de un porcentaje significativo de hierbas y arbustos en dos de ellas, mientras que la población de la tercera era baja, alrededor del $1 \%$ del suelo. La parcela seleccionada como de resistencia regenerativa media tenía una cubierta vegetal inferior que la de alta resistencia. Este dato generó muchas discusiones y hubo que postergar la decisión al respecto para cuando se desarrollaran las conclusiones.

2.2. Diagrama explicativo del Método de intercepción en un rango de vegetación aleatorio aplicado en cada una de las tres parcelas para el estudio de la cubierta vegetal.

2.3. Las cantidades de magnesio, calcio y potasio (nutrientes esenciales) eran más altas en parcelas con resistencia media o alta que en la parcela con baja. A falta de confirmación en las conclusiones, se consideró como hipótesis que los valores más altos de caliza activa en las parcelas de resistencia media y alta implicaría una posible deficiencia de hierro (nutriente esencial de las plantas) (Lindsay 1984).

2.4. En el estudio de la composición del suelo, el porcentaje de arena, limo y arcilla mostraba que era muy similar en las tres parcelas. Franca para parcelas de baja y alta resistencia y franco-arenosa en la media.

Es importante resaltar que los resultados de la cobertura vegetal en las parcelas media y baja parecían contradecirse entre sí. El grupo encargado de realizar este cartel dudó mucho sobre su inclusión, puesto que parecían refutar las hipótesis de investigación. Como no alcanzaron ningún acuerdo se decidió aplazar la discusión y presentar el problema a los otros grupos para conocer su opinión al respecto.

En cuanto a la teledetección (figura 10), se les pidió que estimaran tanto la información global del área de estudio, como a nivel local para cada una de las distintas parcelas. El estudio local se consiguió mediante Landsat, pues el tamaño de su resolución $(30 * 30 \mathrm{~m})$ coincide con el de las parcelas estudiadas. Además, se les explicó qué es la recta de regresión (Matemáticas) para una nube de puntos; la cual se empleó para evaluar la distribución de los datos por encima o debajo de la media del NDVI. Así, los aspectos considerados fueron los siguientes:

3.1. Se indicó, brevemente, en qué consiste el índice NDVI. 
3.2. A través de los valores NDVI obtenidos mediante Landsat, tras eliminar el ruido (cantidades negativas), se obtuvo un $9 \%, 52 \%$ y $57 \%$ de disminución para las parcelas de baja, media y alta resistencia, respectivamente. La recta de regresión asociada a los valores obtenidos representaba un valor promedio (no se consideraba una línea de tendencia).

3.3. Los datos Sentinel indicaban claramente cuándo se produjo el incendio. Los valores NDVI son estacionales, pero en los últimos 5 años no había habido otro cambio tan abrupto; recogido en un video generado mediante Google Earth (https://cutt.ly/yrtl4Y0).

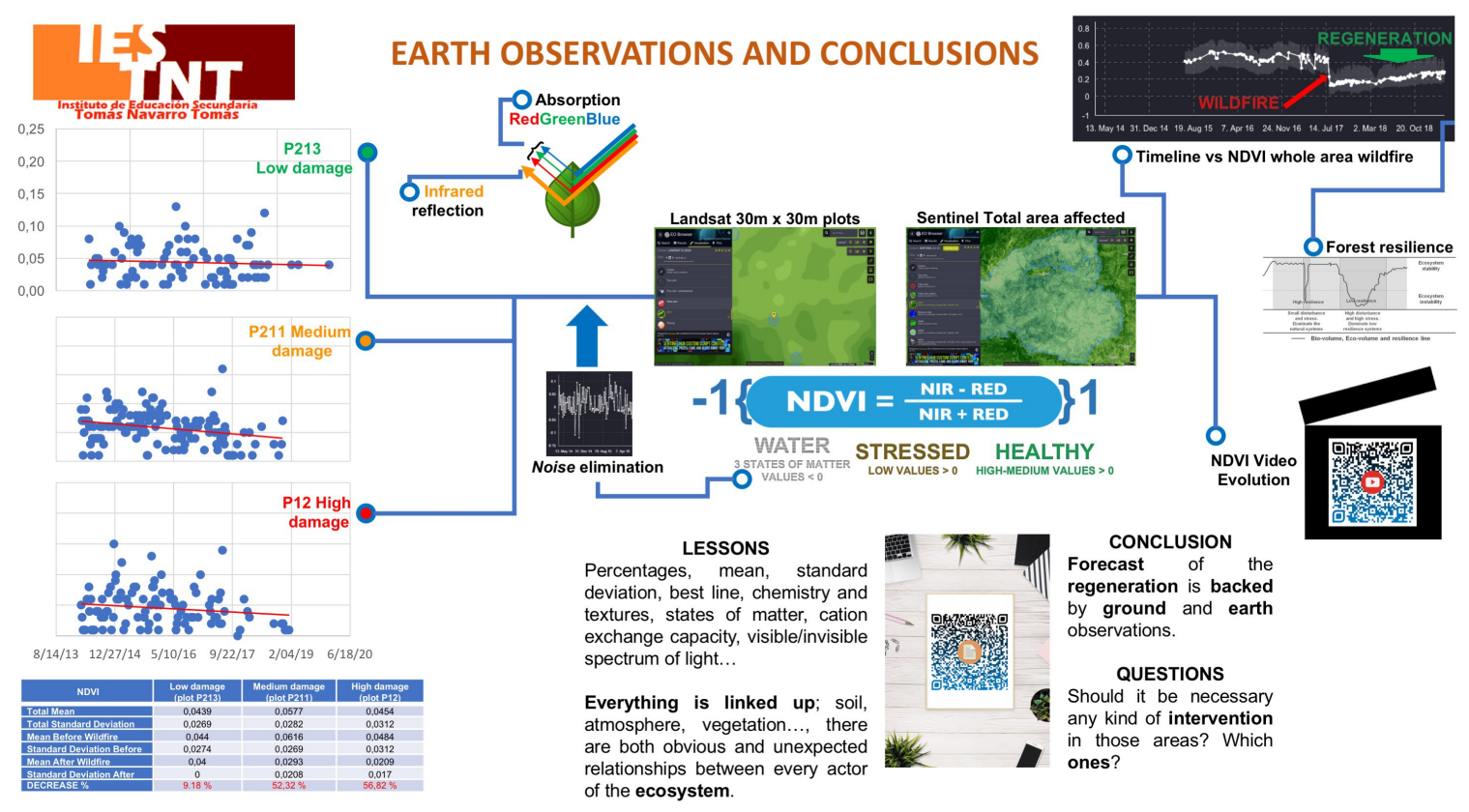

Figura 10. Observación de la tierra y conclusiones finales (Cartel 3 del informe final).

Terminada la recopilación de datos e interpretación de resultados, las últimas sesiones se dedicaron a elaborar las conclusiones y la propuesta para reducir el problema detectado. Para acordar las conclusiones se formularon las siguientes preguntas: ¿Los resultados obtenidos respaldan la predicción del mapa de resistencia regenerativa obtenido en un principio?, ¿por qué? La pizarra fue clave en esta fase. Las intervenciones del alumnado debían ser acompañadas mediante un diagrama o una figura. Muchas ideas eran contradictorias y representaron un reto para llegar a un acuerdo general. Aquí fue fundamental tanto la labor mediadora del profesorado como la charla inicial sobre el trabajo en equipo. Se hizo ver la utilidad de la mediación y de la crítica constructiva; no solo ejerciéndolas, también asumiéndolas. Esto permitió encontrar errores y enriqueció el producto final.

Por ejemplo, una corriente de opinión consideraba incorrecta la predicción inicial del mapa de resistencia regenerativa. Se basaban en que los datos de vegetación de la parcela catalogada como media (P211) tenía porcentajes inferiores a la alta (P12). Pero otro grupo rastreó en los datos previos y comprobó que el punto de partida de la parcela P211 (media), en cuanto a porcentajes de vegetación, tenía valores inferiores respecto a P12 (alta). En otras palabras, la parcela con alta resistencia regenerativa estaba en mejores condiciones que la media, aunque posteriormente la evolución de la media casi había alcanzado los valores de la alta en un año, aproximadamente, de modo que la regeneración en la media había sido mejor.

La recta de regresión ayudó a visualizar que es posible encontrar, entre los últimos valores registrados, algún valor por encima de la recta de regresión para la parcela media, mientras que todos los valores de la baja están por debajo y no se apreciaban indicios de que fuera a mejorar 
como la media (figura 10). Esto fue crítico, pues apoyó la predicción realizada con cierto margen de error. Además, fue útil para discutir resultados en base a datos y no en opiniones subjetivas.

Por último, la mejora que debía proponerse giró en torno a la cuestión de si es o no conveniente actuar tras un incendio. Los resultados evidenciaban una región de muy difícil recuperación y cuyas causas, posiblemente, se debieran a una alta erosión causada por la pendiente y lluvias torrenciales. Quizá, si en este tipo de regiones se hubiera actuado inmediatamente después del incendio, recubriendo el suelo con paja o resembrando especies autóctonas, por ejemplo, los resultados hubieran sido diferentes. De hecho, hay un debate en el seno de la comunidad científica al respecto; mientras que ciertos autores recomiendan actuar (Ruiz-Gallardo et al 2004), otros no lo hacen (DellaSala y Hanson 2015). El alumnado pudo comprobar que la investigación científica está en constante cambio. Surgen preguntas que son respondidas total o parcialmente, y sus posibles soluciones generan otras cuestiones mucho más difíciles de resolver, es decir, la ciencia está en continuo desarrollo.

Por medio de pactos alcanzados, a partir de los datos recopilados, se acordaron las conclusiones finales, se generaron las versiones definitivas de los carteles y se redactó un informe final para el certamen Climate Detectives.

Para participar en el concurso también se debía entregar un vídeo que no superara los tres minutos de duración como síntesis (accesible vía https://youtu.be/YAgY5Ls71Xs), con el requisito de ser lo más sintético y directo posible a la hora de transmitir las ideas: compresión es comprensión. El guión se acordó durante una sesión en la que tuvo lugar una lluvia de ideas. En el video se incluye la escena de la evolución del NDVI (Google Earth Pro), así como otras en las que el alumnado explica parte de los resultados obtenidos. Algunas intervenciones habían sido rodadas durante el Día de la Ciencia del IES Tomás Navarro Tomás.

El informe final fue registrado en la web ${ }^{18}$ de Climate Detectives el día 15 de abril de 2019.

\section{Resultados del informe final}

De los 118 equipos que participaron en la edición 2019 del certamen escolar, Climate Detectives, dirigido a alumnado de todos los Estados miembros de la ESA, se presentaron a la fase final 30 equipos de ocho países europeos. Un tribunal compuesto por expertos en educación y observación de la Tierra seleccionó cuatro proyectos ganadores que se presentaron en el Living Planet Symposium 2019 de Milán. Los proyectos fueron evaluados en función de su relación con el clima, los datos empleados, su corrección científica, claridad y exhaustividad. Hubo cinco equipos más que obtuvieron una mención de honor, entre ellos, el equipo Climate Quixotes, que participaba con el proyecto comentado en este artículo (ESA 2019b).

\section{Reflexión sobre los resultados de la experiencia}

El estudio de la regeneración de una zona incendiada ha permitido desarrollar algunos aspectos que consideramos muy importantes en el trabajo escolar.

1) El alumnado ha conseguido desarrollar una serie de competencias STEM claves relacionadas con las asignaturas implicadas (Matemáticas, Biología y Geología, Física y Química, Geografía e Historia y Tecnología) como, por ejemplo, el empleo de medios tecnológicos, la recogida y visualización de datos, su posterior análisis o la toma de decisiones sobre la base de criterios racionales.

\footnotetext{
${ }^{18}$ https://climatedetectives.esa.int.
} 
2) Resaltar que, dentro de dichas habilidades STEM, el alumnado adquirió habilidades y metodologías típicas de la investigación científica. En esta dirección, el trabajo conjunto con la Universidad de Castilla-La Mancha también sirve para establecer puentes entre la educación secundaria y la universitaria. Entre el alumnado se despiertan, en cierto sentido, vocaciones científicas.

3) El trabajo en equipo permitió comprender que el avance del conocimiento depende de la colaboración entre iguales, así como desarrollar algunas dinámicas sobre resolución de conflictos mediante consensos.

4) La convergencia en un proyecto común permitió descubrir relaciones y vínculos que mantienen diferentes disciplinas en su tarea de hallar soluciones a problemas que afectan a la vida. Destacar que la temática específica del proyecto, directamente relacionada con el cambio climático, mejoró el nivel de conciencia medioambiental del alumnado, en la medida en que han descubierto las numerosas interrelaciones entre diferentes variables que están afectando al futuro inmediato del planeta.

5) En nuestra opinión, el éxito de la implementación de un proyecto de estas características depende fundamentalmente de las siguientes variables:

a) La formación del profesorado en aspectos concretos relacionados con el proyecto y, sobre todo, el compromiso alcanzado, que facilitó una importante implicación en todas las actividades y un adecuado nivel de coordinación.

b) La labor vertebradora del coordinador sustentada en dos hechos claves: i) ser un formador híbrido, i.e., una figura que aunaba el docente universitario y el profesor de secundaria, y ii) poseer una robusta formación en competencias STEM.

c) La participación del equipo directivo y de las familias, que sirvió para mejorar y optimizar los recursos propios del centro.

d) La incorporación de recursos y la actuación de agentes externos como, por ejemplo, la Universidad de Castilla-La Mancha, necesarios para algunas actividades concretas.

Finalmente, hay que señalar la necesidad de mejorar algunos aspectos organizativos. En este caso, los dos grupos de 12 y 13 años no tenían horas en común que pudieran emplearse de forma sistemática. Este aspecto debió considerarse con anterioridad a la puesta en práctica de la actividad, de modo que los grupos involucrados deberían haber tenido una sesión que coincida en el horario y, si es posible, para una misma materia involucrada en el proyecto. Dentro de la necesaria planificación académica de los centros educativos, habría que valorar también la posibilidad de flexibilizar aquellos aspectos que permitan introducir mejoras educativas y proyectos de innovación docente.

\section{Agradecimientos}

Agradecemos el decidido e inapelable compromiso del profesorado implicado en el proyecto del IES Tomás Navarro Tomás de Albacete, así como a la Universidad de Castilla-La Mancha por su apoyo logístico y formativo a lo largo de toda la experiencia, en particular a la ETSIAM y a la Facultad de Educación de Albacete. También agradecemos la ayuda económica concedida por el Ayuntamiento de Albacete que permitió sufragar los análisis de suelo realizados. Asimismo, reconocemos las excelentes iniciativas educativas dirigidas por la Oficina Europea de Recursos para la Educación Espacial (ESERO) entre las que se encuentra el certamen Climate Detectives.

\section{Referencias}

AEMET (2019a) Generación de escenarios regionalizados de cambio climático. Recuperado de https://cutt.ly/MsfRG3s

AEMET (2019b) Regionalización AR5-IPCC. Gráficos de evolución. Regionalización estadística análogos. Albacete. Recuperado de https://cutt.ly/4rtfRHJ 
Asadnabizadeh M. (2019) Development of UN Framework Convention on Climate Change Negotiations under COP25: Article 6 of the Paris Agreement perspective, Open Political Science, 2(1), 113-119. https://doi.org/10.1515/openps-2019-0012

Balgopal MM. (2020) STEM teacher agency: A case study of initiating and implementing curricular reform. Science Education. 104:762-785. https://doi.org/10.1002/sce.21578

Canfield R. (1941) Application of the Line Interception Method in Sampling Range Vegetation. Journal of Forestry 39, 388-349.

Cebrián, G., Junyent, M. (2015) Competencies in Education for Sustainable Development: Exploring the Student Teachers' Views. Sustainability, 7, 2768-2786.

Couso D. (2017) Perque estem a STEM? Definint l'alfabetitzacio STEM per a tothom i amb valors. Revista Ciències, 34, 21-29.

DellaSala D. A. y Hanson Ch. T. (2015) The Ecological Importance of Mixed-Severity Fires:Nature's Phoenix. New York: Elsevier.

Domènech-Casal J. (2014) Contextos de indagación y controversias socio-científicas para la enseñanza del Cambio Climático. Enseñanz̧a de las Ciencias de la Tierra, 22(3), 267-276.

Domènech-Casal J., Lope S., y Mora Ll. (2019) Qué proyectos STEM diseña y qué dificultades expresa el profesorado de secundaria sobre el Aprendizaje Basado en Proyectos. Revista Eureka sobre Enseñanza y Divulgación de las Ciencias 16(2), 2203. doi: 10.25267/Rev_Eureka_ensen_divulg_cienc.2019.v16.i2.2203

European Comission (2015) Science Education for Responsible Citizenship. Luxembourg: Publications Office of the European Union. doi:10.2777/12626.

ESA (2019a) More of Africa Scarred by Fires than Thought. ESA Applications. Recuperado de https://cutt.ly/psfRseK

ESA (2019b) LPS 2019 Climate Detectives School Award: and the winners are... ESA Agency. Recuperado de https://cutt.ly/YrhI147

Falloon G., Hatzigianni M., Bower M. Forbes A. y Stevenson M. (2020) Understanding K-12 STEM Education: a Framework for Developing STEM Literacy. J Sci Educ Technol 29, 369-385. https://doi.org/10.1007/s10956-020-09823-x

García R. y Báez A. P. (2012) Atomic Absorption Spectrometry (AAS). En M. AkhyarFarrukh (Ed.), Atomic Absorption Spectroscopy (pp. 1-13). London: IntechOpen. https://cutt.ly/krkRbtE

Garcia-Piqueras M. (2017) Kolmogórov: la dualidad entre el caos y la determinación. Barcelona: RBA.

Geoghegan L., Dulewicz V. (2008). Do Project Managers' Leadership Competencies Contribute to Project Success. Project Management Journal, 39(4), 58-67.

Heckman J., Kautz T. (2012) Hard evidence on soft skills. Labour Economics, 19(4), 451464.

Holmlund T., Lesseeig K., Slavit D. (2018) Making sense of STEM education in K-12 contexts. International Journal of STEM Education, 5(32), 1-18.

Keeley J. E. (2009) Fire intensity, fire severity and burn severity: A brief review and suggested usage. International Journal of Wildland Fire 18(1), 116-126.

Kilpatrick W.E. (1918) The Project Method: the use of the purposeful act in the educative process. Teachers college, Columbia University. 
King D. (2012) New perspectives on context-based chemistry education: Using a dialectical sociocultural approach to view teaching and learning. Studies in Science Education, 48(1), 51-87.

König B., Diehl K., Tscherning D., Helming K. (2013) A framework for structuring interdisciplinary research management. Research Policy, 42(1), 261-272, ISSN 0048-7333. https://doi.org/10.1016/j.respol.2012.05.006

Larson E. W., Gray C. F. (2014). Project management: The managerial process (6th ed.). New York, NY: McGraw-Hill Education.

Lindsay W. L. (1984) Soil and plant relationships associated with iron deficiency with emphasis on nutrient interactions. Journal of Plant Nutrition, 7:1-5, 489-500. https://cutt.ly/arkTYdv

Leibniz G. W. (2017) Discurso de la metafísica. Madrid: Alianza Editorial.

López V., Couso D., Simarro C., Garrido A., Grimalt-Alvaro C., Hernandez M.I. y Pinto R. (2017) El papel de las TIC en la enseñanza de las ciencias en secundaria desde la perspectiva de la práctica científica. Enseñanza de las Ciencias, número Extraordinario 2017, 691-697.

Montero-Silvestre I. (2018) Severidad e Impacto del Incendio Forestal de Yeste (Albacete) del Verano de 2017 (3200 ha quemadas). Trabajo de Fin de Grado en Ingeniería Forestal y del Medio Natural, Universidad de Castilla-La Mancha, Albacete.

Novo B., Landis E. A., Haley M. L. (2017). Leadership and Its Role in the Success of Project Management. Journal of Leadership, Accountability and Ethics, 14(1).

Pearson T.R.H., Brown S., Murray L., Sidman G. (2017) Greenhouse gas emissions from tropical forest degradation: an underestimated source. Carbon Balance Manage 12, 3. https://doi.org/10.1186/s13021-017-0072-2

Peters H. (2015) Game theory: A Multi-leveled approach. Berlin: Springer-Verlag.

Potvin P., Riopel M., Masson S., Fournier F. (2010) Problem-centered learning vs. teachingcentered learning in science at the secondary level: An analysis of the dynamics of doubt. Journal of applied research on learning, 3, 1-24. https://cutt.ly/brhAL4R

Quigley C.F., Herro D. (2016) "Finding the Joy in the Unknown": Implementation of STEAM Teaching Practices in Middle School Science and Math Classrooms. J Sci Educ Technol 25, 410-426. https://doi.org/10.1007/s10956-016-9602-z

Quigley C.F., Herro D., King E., Plank, H. (2020) STEAM Designed and Enacted: Understanding the Process of Design and Implementation of STEAM Curriculum in an Elementary School. J Sci Educ Technol. https://doi.org/10.1007/s10956-020-09832-w

Quinn R. E., Rohrbaugh J. (1983) A spatial model for effectiveness criteria: towards a competing values framework to organisational analysis. Management Science 29, 363-377.

Quinn R. E. (1988) Beyond Rational Management: Mastering the Paradoxes and Competing Demands of High Perfomance. Jossey-Bass. San Francisco.

Ruiz-Gallardo J. R., Castaño S. y Calera A. (2004) Application of remote sensing and GIS to locate priority intervention areas after wildland fires in Mediterranean systems: a case study from south-eastern Spain. International Journal of Wildland Fire 13(3), 241-252.

Sanmartí N. y Márquez C. (2017) Aprendizaje de las ciencias basado en proyectos: del contexto a la acción. Ápice, Revista de Educación Científica, 1(1), 3-16. 
Sanmartí N. (2019) ¿Es posible una evaluación gratificante y útil para aprender? Uno 86, 43-49.

Sanmartí N. (2020) Evaluar y aprender: un único proceso. Barcelona: Octaedro.

Schmid B., Adams J. (2008). Motivation in project management: The project manager's perspective. Project Management Journal, 39(2), 60-71.

Slough S.W., Milam J.O. (2013) Theoretical Framework for the Design of STEM ProjectBased Learning. In: Capraro R.M., Capraro M.M., Morgan J.R. (eds) STEM ProjectBased Learning. Sense Publishers, Rotterdam.

Weier J. y Herring D. (2000) Measuring Vegetation (NDVI and EVI), The Earth Observatory, NASA. https://go.nasa.gov/2PRlkZN 